Published in final edited form as:

Leuk Res. 2016 February ; 41: 43-47. doi:10.1016/j.leukres.2015.12.007.

\title{
Outcomes of patients with myelodysplastic syndromes who achieve stable disease after treatment with hypomethylating agents
}

\author{
Aziz Nazha ${ }^{a}$, Mikkael A. Sekeres ${ }^{a}$, Guillermo Garcia-Manero ${ }^{b}$, John Barnard ${ }^{c}$, Najla H. Al \\ Ali $^{d}$, Gail J. Roboz ${ }^{e}$, David P. Steensma ${ }^{f}$, Amy E. DeZern ${ }^{9}$, Cassie Zimmerman ${ }^{a}$, Elias J. \\ Jabbour $^{b}$, Katrina Zellc ${ }^{c}$, Alan F. List ${ }^{d}$, Hagop M. Kantarjian ${ }^{b}$, Jaroslaw P. Maciejewski ${ }^{\mathrm{a}}$, and \\ Rami S. Komrokjid, ${ }^{\star}$ On behalf of the: MDS Clinical Research Consortium \\ aLeukemia Program, Cleveland Clinic, Taussig Cancer Institute, Cleveland, $\mathrm{OH}$, United States \\ ${ }^{b}$ Department of Leukemia, The University of Texas MD Anderson Cancer Center, Houston, TX, \\ United States \\ 'Department of Quantitative Health Sciences, Cleveland Clinic, Cleveland, OH, United States \\ ${ }^{\mathrm{d} D e p a r t m e n t}$ of Malignant Hematology, H. Lee Moffitt Cancer Center \& Research Institute, \\ Tampa, FL, United States \\ eDivision of Hematology and Oncology, New York Presbyterian Hospital-Weill Cornell Medical \\ College, New York, NY, United States \\ fDepartment of Medical Oncology, Dana-Farber Cancer Institute, Harvard Medical School, \\ Boston, MA, United States \\ 9Division of Hematologic Malignancies of Oncology, The Johns Hopkins University School of \\ Medicine, Baltimore, Maryland, United States
}

\begin{abstract}
Treatment with hypomethylating agents (HMAs) improves overall survival (OS) in patients who achieve a response of stable disease (SD) or better (complete remission [CR], partial remission [PR], or hematologic improvement [HI]). It is not well established if patients who achieve SD at 4-6 months of therapy should be offered different therapies to optimize their response or continue with the same regimen. Clinical data were obtained from the MDS Clinical Research Consortium database. SD was defined as no evidence of progression and without achievement of any other responses. Of 291 patients treated with AZA or DAC, 55\% achieved their best response (BR) at 46 months. Among patients with SD at 4-6 months, 29 (20\%) achieved a better response at a later
\end{abstract}

\footnotetext{
* Corresponding author at: Department of Hematologic Malignancies, H. Lee Moffitt Cancer Center and Research Institute, 12902 Magnolia Dr, Tampa, FL 33612, United States. Rami.komrokji@moffitt.org (R.S. Komrokji).

Conflict of interest

The authors disclose no potential conflicts of interest.

Contributions

A.N., M.A.S., and R.S.K. designed the study, analysed and interpreted the data, wrote and approved the manuscript. N.A., J.B., K.Z., C.Z., and M.G. collect the data and performed the statistical analyses. G.G.M., G.R., D.S., A.D., E.J., A.L, H.K., and J.P. analysed the data reviewed and approved the manuscript.
} 
treatment time point. Younger patients with lower bone marrow blast percentages, and intermediate risk per IPSS-R were more likely to achieve a better response (CR, PR, or HI) after SD at 4-6 months. Patients with SD who subsequently achieved CR had superior OS compared to patients who remained with SD (28.1 vs. 14.4 months, respectively, $p=.04$ ). In conclusion, patients treated with HMAs who achieves CR after a SD status had longer survival with continuous treatment after 6 months.

\section{Keywords}

MDS; Response; Stable disease; Hypomethylating agent

\section{Introduction}

Patients with higher-risk myelodysplastic syndromes (MDS) have a high likelihood of transforming to acute myeloid leukemia (AML) and an overall survival (OS) measured in months. The primary goal of therapy in these patients is to alter the natural course of the disease [1-3]. The DNA methyltransferase inhibitors azacitidine (AZA) and decitabine (DAC) are considered first-line therapies, with AZA having demonstrated an improvement in overall survival compared to conventional care regimens, at a median of 24.5 months vs. 15.0 months, respectively, in the AZA-001 study [4, 5]. This impact on survival was observed in AZA-treated patients despite relatively low response rates (complete remission (CR) 17\% and partial remission (PR) 12\%) [6]. A subsequent analysis of the AZA-001 trial showed that treatment with AZA can prolong OS even in patients who did not achieve a CR or PR [7], raising the question of whether achieving a CR should be a therapeutic goal [8]. Furthermore, AZA-treated patients achieving a hematologic improvement (HI) or better had a 95\% reduction in the risk of death compared to patients treated with conventional care (hazard ratio.05 [95\% CI:.01-.43], $P=.006$ ) [9].

The decision of when to continue higher-risk MDS patients on AZA or DAC to maximize their chance of response, or of concluding that a response is unlikely to occur and switching to another agent, has been a challenge to address. In the AZA-001 trial, the median number of cycles to first response was three (range: 1-22); $81 \%$ of patients achieved a first response by six cycles, and $90 \%$ achieved a first response by nine cycles, suggesting that a median of 9 cycles of treatment is needed to realize the majority of responses [10]. In a subsequent analysis of the AZA-001 trial, 19\% of patients who achieved stable disease (SD) as their best response to AZA at three months achieved a better response $\mathrm{HI}+(\mathrm{CR}, \mathrm{PR}$, or $\mathrm{HI})$ at six months, while only $14 \%$ of patients with SD at six months achieved a better response by 9 months [9]. The outcome of patients who had SD on AZA therapy was similar to patients who received conventional care treatment while patients who achieved HI+ on AZA therapy had better outcome compared to those achieving HI+ on conventional care at any time point. Similarly, in a randomized, phase III trial of low dose decitabine versus best supportive care, 16 of 119 patients (13\%) who received decitabine achieved CR, 7(6\%) a PR, and 18 (15\%) achieved HI [11]. Median time to best response was 3.8 months (range, 1.4-11.8 months) for all responders, with a median of 5.8 months to reach $\mathrm{CR}, 2.9$ months for PR, and 3.8 months for HI [11]. It is thus not well established if patients who achieve SD by 6 months of therapy 
with HMAs should be offered different therapies to optimize their response, or continue with the same HMA regimen.

Here we compared the outcomes of patients who achieved SD to AZA or DAC as their best response (BR) to those achieving better responses. We also explored whether patients who achieve SD at 4-6 months of therapy and subsequently achieve a better response had improved outcomes compared to patients who achieve only SD as their best response at any time point during therapy.

\section{Methods}

\subsection{Patients}

Patient data from the MDS Clinical Research Consortium institutions (Moffitt Cancer Centre $n=259$, Cleveland Clinic $n=221$, MD Anderson Cancer Centre $n=192$, Cornell University $n=100$, Dana-Farber Cancer Institute $n=45$, and Johns Hopkins $n=29$ ) were included. Patients were diagnosed with MDS (according to 2008 WHO criteria and confirmed at each participating institution) and had higher-risk disease by the International Prognostic Scoring System (IPSS) or the revised IPSS (IPSS-R) [12]. All patients were treated with either AZA or DAC for 5-7 days of 28-day cycles. All data collected from each institution were stored and secured in an IRB approved database at Cleveland Clinic.

\subsection{Responses and outcome}

Response definitions, including CR, PR, HI, SD, and progressive disease (PD) were defined per International Working Group (IWG) 2006 criteria [8]. Responses were characterized as initial response (IR) and BR. IRs were defined as responses after 4-6 cycles of treatment with either AZA or DAC. BR was defined as the best response achieved by a patient at any time point after or including IR. For example, if a patient achieved SD after 4-6 cycles of treatment and then achieved an HI thereafter, that patient's IR would be SD and BR would be HI.

\subsection{Statistical analysis}

Overall survival (OS) was calculated from time of initiation of treatment to time of death or last follow up. Leukaemia-free survival was calculated from the time of treatment initiation to time of AML transformation. Differences among variables were evaluated by the Chi Square and Mann-Whitney $U$ test for categorical and continuous variables among patient groups, respectively. Time-to-event analyses were performed by the Kaplan-Meier method, and survival curves were compared using the 2 -tailed log rank test. A two-sided $P$ value $\leq 05$ was considered to be statistically significant.

\section{Results}

\subsection{Patient characteristics}

Of 846 patients with MDS treated with AZA or DAC, we identified 291 higher-risk patients who had response data documented at each time point (initial and best response) and met our inclusion criteria. Patient characteristics are summarized in Table 1. The median age was 70 
years (range, 35-99), 248 patients (85\%) received treatment with AZA and $43(15 \%)$ with DAC. IPSS risk categories included 65 patients (22\%) who were intermediate-1, 173 (59\%) intermediate-2, 47 (16\%) high, and 6 (2\%) not assessable (missing values). Per IPSS-R, 58 patients (20\%) were intermediate risk, 107 (37\%) high, 126 (43\%) very high, and $6(2 \%)$ not assessable. A total of 142 patients (49\%) progressed to AML. Patients received a median of 6 cycles of AZA or DAC (range, 4-52). Median time from date of diagnosis to start of treatment was 1 month (range, 0-53).

\subsection{Responses at each time point}

Patients had an IR of: CR in 28 patients (10\%), PR in 15 (5\%), HI in 29 (10\%), SD in 144 (49\%), and progressive disease (PD) in $75(26 \%)$ for an initial overall response rate (CR + $\mathrm{PR}+\mathrm{HI}$ ) of 25\%. Response rates improved when considering BR: CR in 47 (16\%), PR in 19 (7\%), HI in 35 (12\%), SD in 113 (39\%), and PD in 77 (26\%) for a best overall response rate of 35\%. Among responders, 181/214 (85\%) achieved their response at months 4-6 and continued to have a similar response for the remainder of their treatment. Two patients with PR as their IR subsequently achieved CR and two patients with HI subsequently achieved PR and CR, respectively, with a median time form IR to BR of 5.1 months (range, 2.6-27.3). Among the 144 patients who had SD as their IR, 29 (20\%) achieved a better response at a later treatment time point (16 [55\%] CR and 13 [45\%] PR/HI), with a median time from IR to BR of 3.7 months (range, 1.2-14.5); 113 (78\%) continued to have stable disease, and 2 (1\%) progressed to AML. Younger patients (median age 67 vs. 70 years, $p=.02$ ) with lower WBCs (a median of 2.5 vs. 2.9, $p=.06$ ), lower bone marrow blast percentages (median of $7 \%$ vs. $10 \%, p=.05)$ and intermediate risk per IPSS-R ( $41 \%$ vs. $19 \%, p=.01)$ were more likely to achieve a better response (CR, PR, or HI) after SD at 4-6 months compared to patients remaining in SD (Table 2).

\subsection{Survival and AML transformation}

With a median follow up of 16.5 months (range, 2.5-120.2), the median OS for the entire group was 15.5 months (range, 2.5-120). Median OS from start of therapy based on BR was: 19.7, 12.6, 15.4, 13.7 and 10.1 months for CR, PR, HI, SD, and PD, respectively, Fig. 1 A. Median OS for from the response at 4-6 months to last follow up or death was 15.5, 7.6, 9.2, 10.6, and 6.3 months for CR, PR, HI, SD, and PD, respectively. Patients who achieved a BR of CR, PR, HI, or SD had a longer OS compared to patients with PD $(p<.001)$ for each response, Fig. 1A. Although patients who achieved CR as their BR had a greater median OS compared to patients achieving PR (19.7 vs. 12.6 months, $p=.29$ ), HI (19.7 vs. 15.4 months, $p=.16$ ), and SD (19.7 vs. 13.7 months, $p=.13$ ), respectively, differences were not statistically significant, Fig. 1A Patients with SD as their IR who subsequently achieved CR had superior OS compared to patients who remained with SD (28.1 vs. 14.4 months, respectively, $p=.04$ ), while patients who subsequently achieved PR or HI following SD had a similar survival compared to patients continuing to have SD (12.1 vs. 14.4 months, respectively, $p=.81$ ). This survival benefit remained significant when survival was calculated from the time of BR to time of last follow up or death, with patients achieving a CR having a median OS of 24.5 months, vs. 9.9 months for patients with SD, $p<.01$, Fig. 2. 
A total of 142 patients (49\%) progressed to AML, with a median time to AML progression from start of therapy of 14.6 months (range,.6-75.3). The median time to AML transformation from starting treatment was longer among patients who achieved CR as their $\mathrm{BR}$, at 17.8 months, compared to patients who achieved PR at 16.3 months, HI, at 13.3 months, and SD, at 10.0 months, Fig. 1B.

\section{Discussion}

Patients with higher-risk MDS have significantly poor overall survival and limited therapeutic options. Standard, front-line therapy involves the use of HMAs: either AZA or DAC. In the absence of frequent bone marrow biopsies, it often is challenging to determine whether or not a patient is responding to therapy, particularly in the setting of cytopenias that may be related to underlying disease and/or to therapy. Furthermore, it is a clinical challenge to decide whether or not to continue a patient when the response may not be definitive.

In this study, we evaluated the clinical outcome of patients with MDS who were treated with AZA or DAC based on their response to treatment, per IWG 2006 criteria [8]. Patients who achieve any response, including $\mathrm{CR}, \mathrm{PR}, \mathrm{HI}$, and $\mathrm{SD}$, live significantly longer than those who have PD, with those achieving CR demonstrating the longest median survival. Additionally achievement of any response, even if the definition of "response" included SD, as BR delayed AML transformation compared to patients with PD. Analyses of randomized trials of HMAs have compared the outcome of patients with MDS who received either AZA or DAC to those receiving conventional care, including best supportive care or low/highdose chemotherapy $[5,6,10,11]$. In these trials, patients treated with HMAs clearly achieved higher response rates compared to patients in conventional care arms. More importantly, and in multivariate analysis, SD status was associated with a $91 \%$ reduction in risk of death regardless of whether the patients received AZA or conventional care [9]. Our study is the first to compare responses entirely within a group of patients who all received HMAs, and we similarly showed improved survival for patients who had SD in response to therapy, suggesting that SD remains a valid response definition, and a valuable clinical determinant to consider continuation of therapy.

Another important clinical question is the duration of treatment with HMA and whether patients who achieved a SD as their initial response should be continued on the same treatment or be offered different therapy. In a secondary analysis of AZA-001, Silverman et al. showed that the median time to first response with AZA was 2 months (range, 1-16) [10]. Overall, $91 \%$ of responding patients achieved their first response within 6 cycles. Of the remaining $9 \%$ of patients, all achieved their first response by 12 cycles, except 1 patient who had a first response at cycle 16 [10]. In our analyses, the median time form IR to BR was 5.1 months (range, 2.6-27.3). Most patients (85\%) achieved their BR at 4-6 cycles, with the remainder $(15 \%)$ achieving a better response with continuous treatment. Further, $20 \%$ of patients with SD at 4-6 months achieved a BR when their treatment continued. More importantly, patients who achieved CR after an IR of SD had increased overall survival compared to patients who remained in $\mathrm{SD}$, suggesting that $\mathrm{CR}$ remains an important endpoint for treating MDS patients. Further, patients with a lower likelihood of achieving the goal of a BR of CR can be identified. Those with a higher risk category per IPSS-R, and 
with a higher blast percentage are unlikely to improve on their SD response, and should be offered a different treatment strategy in an attempt to improve the quality of their response. This strategy could be achieved by enrolling these patients into clinical trial that add a second drug. However, in the abscess of an available clinical trial, treatment with HMA should be continued.

Patients included in this study were treated with HMAs at MDS specialty centres, but not routinely as part of an interventional clinical trial. Thus, the median OS for our patient cohort was lower than the OS reported from the AZA-001 trial but, it is closer to the median OS reported from the CLGB trial which randomized patients to receive azacitidine at standard dose versus supportive care and showed median OS of 18 months in patients treated with azacitidine compared to 11 months for those treated with supportive care [4]. This likely represents differences in treatment patterns in different parts of the world and subtle eligibility differences.

It is possible that patients who have SD after 4-6 cycles may have biologically less aggressive disease than those who experience early progression, which could theoretically lead to a better outcome regardless of therapeutic intervention. Patients such as this are typically continued on HMA therapy if they are tolerating the drug without significant toxicity. We caution against concluding that SD should signify that the HMA is the ultimate therapy for these patients, but suggest this allows for more time to pursue evaluation of additional therapies to improve the response beyond the SD. However, one strength of this study is the demonstration that a significant minority of these patients (20\%) had their BR with more than 4-6 cycles and it is these patients who ultimately survived longer than patients who only ever achieved SD.

In conclusion, the majority of patients treated with HMAs will achieve their BR by 4-6 months of treatment, but a substantial fraction will achieve a BR with additional treatment. Patients who achieved CR after a SD status at 4-6 months had a longer survival than those who maintained their SD status, suggesting that $\mathrm{CR}$ remains an important treatment goal, and evaluation of response at different time points such as at 3,6, and 9 months of therapy with HMA may also be important to address in future studies. A new strategies to improve the chance of better response - either by adding a second drug or switching therapy in patients with $\mathrm{SD}$ - should be also explored.

\section{Acknowledgments}

M.A.S., G.G.M., G.J.R., D.P.S., A.E.D., C.Z., S.L., J.P.M., and R.S.K. all are grateful to receive support from the Edward P. Evans Foundation.

\section{References}

1. Sekeres MA, Cutler C. How we treat higher-risk myelodysplastic syndromes. Blood. 2014; 123:829-836. [PubMed: 24363399]

2. Garcia-Manero G. Myelodysplastic syndromes: 2014 update on diagnosis, risk-stratification, and management. Am J Hematol. 2014; 89:97-108. [PubMed: 24464505] 
3. Bejar R, Tiu RV, Sekeres MA, Komrokji RS. Myelodysplastic syndromes: recent advancements in risk stratification and unmet therapeutic challenges. American Society of Clinical Oncology Educational Book/ASCO American Society of Clinical Oncology Meeting. 2013; 2013

4. Silverman LR, Demakos EP, Peterson BL, et al. Randomized controlled trial of azacitidine in patients with the myelodysplastic syndrome: a study of the cancer and leukemia group B. J Clin Oncol. 2002; 20:2429-2440. [PubMed: 12011120]

5. Kantarjian H, Issa JP, Rosenfeld CS, et al. Decitabine improves patient outcomes in myelodysplastic syndromes: results of a phase III randomized study. Cancer. 2006; 106:1794-1803. [PubMed: 16532500]

6. Fenaux P, Mufti GJ, Hellstrom-Lindberg E, et al. Efficacy of azacitidine compared with that of conventional care regimens in the treatment of higher-risk myelodysplastic syndromes: a randomised, open-label, phase III study. Lancet Oncol. 2009; 10:223-232. [PubMed: 19230772]

7. List AF, Fenaux P, Mufti GJ, Hellström-Lindberg E, Gore S, Bennett JM, Silverman LR, Backstrom J, Allen AR, Beach CL. Azacitidine (AZA) extends overall survival in higher-risk myelodysplastic syndromes (MDS) without necessity for complete remission. J Clin Oncol. 2008; 26(155):7006.

8. Cheson BD, Greenberg PL, Bennett JM, et al. Clinical application and proposal for modification of the International Working Group (IWG) response criteria in myelodysplasia. Blood. 2006; 108:419425. [PubMed: 16609072]

9. Gore SD, Fenaux P, Santini V, et al. A multivariate analysis of the relationship between response and survival among patients with higher-risk myelodysplastic syndromes treated within azacitidine or conventional care regimens in the randomized AZA-001 trial. Haematologica. 2013; 98:1067-1072. [PubMed: 23585522]

10. Silverman LR, Fenaux P, Mufti GJ, et al. Continued azacitidine therapy beyond time of first response improves quality of response in patients with higher-risk myelodysplastic syndromes. Cancer. 2011; 117:2697-2702. [PubMed: 21656747]

11. Lubbert M, Suciu S, Baila L, et al. Low-dose decitabine versus best supportive care in elderly patients with intermediate- or high-risk myelodysplastic syndrome (MDS) ineligible for intensive chemotherapy: final results of the randomized phase III study of the European Organisation for Research and Treatment of Cancer Leukemia Group and the German MDS Study Group. J Clin Oncol. 2011; 29:1987-1996. [PubMed: 21483003]

12. Greenberg PL, Tuechler H, Schanz J, et al. Revised international prognostic scoring system for myelodysplastic syndromes. Blood. 2012; 120:2454-2465. [PubMed: 22740453] 

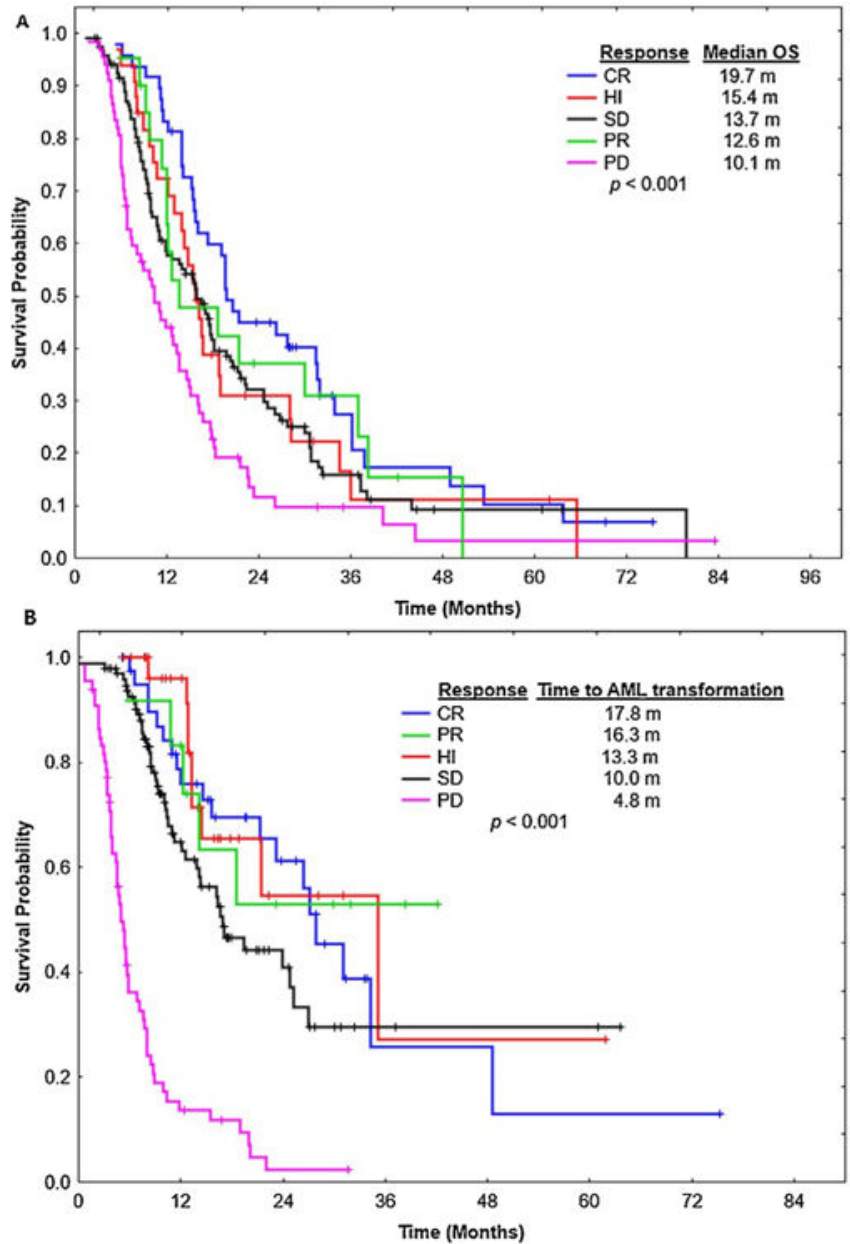

Fig. 1.

Overall survival and Leukaemia-free survival based on best response. (A) shows overall survival based on responses criteria. $\mathrm{CR}=$ complete remission, $\mathrm{HI}=$ hematologic improvement. $\mathrm{SD}=$ stabledisease, $\mathrm{PD}=$ progressive disease, and $\mathrm{PR}=$ partial response. $(\mathrm{B})$ shows time to acute myeloid leukaemia (AML) transformation from start of therapy with hypomethylating agents based on response criteria. 


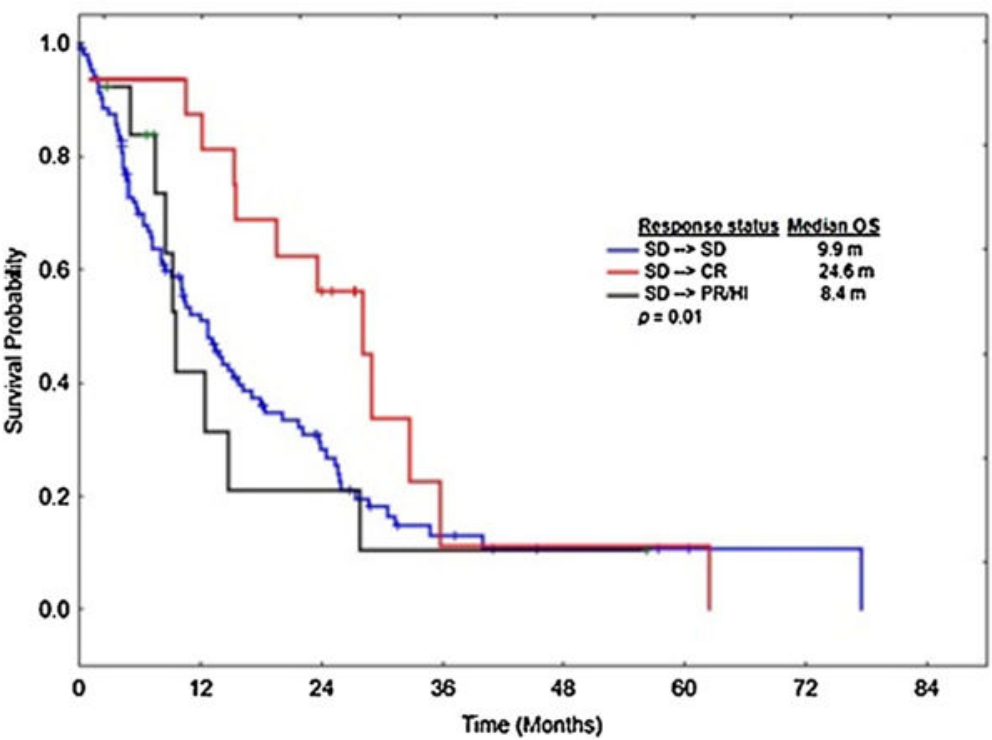

Fig. 2.

Overall survival in patients who achieved $\mathrm{CR}$ as their best response compared to $\mathrm{PR} / \mathrm{HI}$ and SD among patients who had SD at 4-6 months. 


\section{Table 1}

Patient characteristics.

\begin{tabular}{|c|c|}
\hline Parameter & No. $(\%) /[$ range $]$ \\
\hline Total & 291 \\
\hline Median age, years & 70 [35-99] \\
\hline \multicolumn{2}{|l|}{ Gender } \\
\hline Male & $193(66)$ \\
\hline Female & $98(34)$ \\
\hline \multicolumn{2}{|l|}{ Race } \\
\hline White & $259(89)$ \\
\hline African American & $13(4)$ \\
\hline Hispanic & $6(2)$ \\
\hline Others & $13(4)$ \\
\hline \multicolumn{2}{|l|}{ Clinical characteristics } \\
\hline Median white blood cell count $\times 10^{9} / \mathrm{L}$ & $4.80[0.58-68]$ \\
\hline Median hemoglobin, $\mathrm{g} / \mathrm{dl}$ & $9.3[3.7-14.3]$ \\
\hline Median absolute neutrophil count $\times 10^{9} / \mathrm{L}$ & $1.05[0.01-24.8]$ \\
\hline Median platelet $\times 10^{3} / \mathrm{mL}$ & $73[4-659]$ \\
\hline Median bone marrow blast $\%$ & $9[0-21]$ \\
\hline \multicolumn{2}{|l|}{ Cytogenetics by IPSS-R } \\
\hline Very good & $0(0)$ \\
\hline Good & $85(29)$ \\
\hline Inter & $45(15)$ \\
\hline Poor & $60(21)$ \\
\hline Very poor & $95(33)$ \\
\hline Not documented & $6(2)$ \\
\hline \multicolumn{2}{|l|}{ IPSS-R risk category } \\
\hline Intermediate & $58(20)$ \\
\hline High & $107(37)$ \\
\hline Very high & $126(43)$ \\
\hline Not applicable & $6(2)$ \\
\hline \multicolumn{2}{|l|}{ IPSS risk category } \\
\hline Intermediate-1 & $65(22)$ \\
\hline Intermediate-2 & $173(59)$ \\
\hline High & $47(16)$ \\
\hline Not applicable & $6(2)$ \\
\hline \multicolumn{2}{|l|}{ WHO classifications } \\
\hline RCUD & $5(2)$ \\
\hline RCMD & $30(10)$ \\
\hline RARS & $3(1)$ \\
\hline RAEB-1 & $90(31)$ \\
\hline RAEB-2 & $135(46)$ \\
\hline
\end{tabular}




\begin{tabular}{|c|c|c|}
\hline & Parameter & No. $(\%) /[$ range $]$ \\
\hline & MDS associated with isolated del $(5 q)$ & $2(1)$ \\
\hline & MDS-U & $5(2)$ \\
\hline 官 & MDS/MPN & $13(5)$ \\
\hline & Missing & $10(4)$ \\
\hline
\end{tabular}

Abbreviations: IPSS-R, International Prognostic Scoring System-Revised; IPSS, International Prognostic Scoring System; WHO, World Health Organization; RCUD, refractory cytopenia with unilineage dysplasia; RCMD, refractory cytopenia with multilineage dysplasia; RARS, refractory anemia with ring sideroblasts; RAEB, refractory anemia with excess blasts. 


\section{Table 2}

Clinical characteristics of patients who achieved stable disease status at 4-6 months and subsequently achieved a better response compared to patients who continue to have stable disease as their best response.

\begin{tabular}{|c|c|c|c|}
\hline Parameter & SD-> better responseno. $(\%) /[$ range $]$ & SD as best responseno. $(\%) /[$ range $]$ & $P$ \\
\hline Total & $29(20)$ & $113(80)$ & \\
\hline Median age, years & $67(36-78)$ & $70(41-99)$ & .02 \\
\hline Gender & & & .19 \\
\hline Male & $21(72)$ & $81(72)$ & \\
\hline Female & $8(28)$ & $32(28)$ & \\
\hline \multicolumn{4}{|l|}{ Clinical characteristics } \\
\hline Median white blood cell count $\times 10^{9} / \mathrm{L}$ & $2.5[0.8-12.5]$ & $2.9[0.6-62]$ & .06 \\
\hline Median absolute neutrophil count $\times 10^{9} / \mathrm{L}$ & $0.8[0.1-3.5]$ & $1.1[0.1-24.8]$ & .11 \\
\hline Median hemoglobin, g/dl & $9.5[6.8-14]$ & $9.2[3.7-13.5]$ & .52 \\
\hline Median platelet $\times 10^{3} / \mathrm{mL}$ & $94[6-342]$ & 78 [4-659] & .34 \\
\hline Median bone marrow blasts $\%$ & $7[0-18]$ & $10[0-21]$ & .05 \\
\hline \multicolumn{4}{|l|}{ IPSS-R risk category } \\
\hline Intermediate & $12(41)$ & $22(19)$ & .01 \\
\hline High & $9(31)$ & $46(41)$ & .12 \\
\hline Very high & $8(28)$ & $45(40)$ & .08 \\
\hline
\end{tabular}

Abbreviations: SD, stabledisease, IPSS-R, International Prognostic Scoring System-Revised. 